\title{
Real-World Experience of Patient-Relevant Benefits and Treatment Satisfaction with Apremilast in Patients with Psoriasis: An Analysis of the APPRECIATE Study
}

\author{
Toni Maria Klein · Christine Blome - C. Elise Kleyn · Curdin Conrad • Paul G. Sator • \\ Mona Ståhle $\cdot$ Kilian Eyerich · Marc Alexander Radtke · Christine Bundy • \\ Myriam Cordey · Christopher E. M. Griffiths · Matthias Augustin
}

Received: July 29, 2021 / Accepted: October 4, 2021 / Published online: November 23, 2021

(c) The Author(s) 2021

\section{ABSTRACT}

Introduction: In the real-world APPRECIATE study (NCT02740218), most patients with psoriasis demonstrated notable improvements on disease severity measures and reported clinically meaningful treatment benefits with apremilast. Objective: We aim to further describe patientrelevant needs and benefits and patient satisfaction with apremilast, including subgroup analyses based on patient characteristics.

Supplementary Information The online version contains supplementary material available at https:// doi.org/10.1007/s13555-021-00628-3.

T. M. Klein $(\bowtie) \cdot$ C. Blome · M. Augustin Institute for Health Services Research in Dermatology and Nursing (IVDP), University

Medical Center Hamburg-Eppendorf (UKE),

Hamburg, Germany

e-mail: T.Klein@uke.de

C. E. Kleyn · C. E. M. Griffiths

The Dermatology Centre, NIHR Manchester Biomedical Research Centre, Salford Royal Hospital,

The University of Manchester, Manchester, UK

C. Conrad

Department of Dermatology, Lausanne University

Hospital CHUV and University of Lausanne,

Lausanne, Switzerland

P. G. Sator

Department of Dermatology, Clinic Hietzing,

Vienna, Austria
Methods: APPRECIATE, a multinational, retrospective, cross-sectional study, enrolled patients with chronic plaque psoriasis who started apremilast according to the European label. Patient Benefit Index (PBI; range 0 (no patientrelevant benefit) to 4 (maximum patient-relevant benefit), global PBI score $\geq 1$ indicating minimum patient-relevant benefit and $\geq 3$ indicating high benefit) and nine-item Treatment Satisfaction Questionnaire for Medication (TSQM-9; range 0-100) were assessed $6( \pm 1)$ months after apremilast initiation and summarized descriptively. Relationships between

\section{Ståhle}

Unit of Dermatology, Department of Medicine,

Karolinska Institutet, Stockholm, Sweden

K. Eyerich

Department of Dermatology and Allergy, Technical

University of Munich, Munich, Germany

M. A. Radtke

Dermatologikum Hamburg, Hamburg, Germany

C. Bundy

College of Biomedical and Health Sciences, Cardiff

University, Cardiff, UK

M. Cordey

Amgen Europe GmbH, Rotkreuz, Switzerland 
global PBI and TSQM-9 assessments were analyzed by Pearson correlations.

Results: Of 480 enrolled patients, 347 (72.3\%) had remained on apremilast at $6( \pm 1)$ months; $90.9 \%(300 / 330)$ achieved global PBI score $\geq 1$. Mean (standard deviation) global PBI score was 2.8 (1.2). Higher achievement of global PBI score $\geq 3$ was observed in patients with no prior treatments $(61.1 \%(22 / 36))$ or prior phototherapy $(64.6 \%(42 / 65))$ versus prior conventional systemic $(54.4 \%(100 / 184))$ or biologic $(38.6 \%$ $(17 / 44))$ treatment. Strong correlations were observed between the global PBI score and the TSQM-9 global satisfaction and effectiveness subscale scores.

Conclusion: Patients continuing apremilast for $6( \pm 1)$ months in APPRECIATE reported patient-relevant treatment benefits. Findings suggest that receiving apremilast earlier versus later in treatment management is consistent with greater improvements in patient-relevant treatment outcomes.

Keywords: Apremilast; Psoriasis; Patient Benefit Index; Patient-reported outcomes; Realworld study; Treatment satisfaction

\section{Key Summary Points}

Why carry out this study?

Many patients with psoriasis report being dissatisfied with their current treatment, and a better understanding of patient-relevant treatment benefits may help improve treatment satisfaction and effectiveness.

In the multinational, retrospective, crosssectional APPRECIATE study, most patients who initiated apremilast treatment demonstrated patient-relevant treatment benefits, and additional analysis of factors contributing to treatment benefits and satisfaction with apremilast can be useful to physicians in clinical practice.

\section{What did the study ask?}

This analysis of APPRECIATE evaluated patient-relevant needs and benefits using the Patient Benefit Index (PBI) in patient subgroups based on patient characteristics, as well as the association between patientrelevant benefits and treatment satisfaction.

\section{What were the study outcomes/conclusions?}

Continuing apremilast treatment for $6( \pm 1)$ months was associated with patient-relevant treatment benefits, particularly in patients with no prior treatments and those without prior conventional systemic or biologic treatment; achievement of patient-relevant treatment benefits was associated with greater treatment satisfaction.

\section{What was learned from the study?}

Findings confirm the value of patientrelevant measures such as the PBI to evaluate treatment satisfaction and effectiveness, and suggest that patients may benefit from apremilast treatment earlier rather than later in treatment management.

\section{INTRODUCTION}

Plaque psoriasis is a long-term immune-mediated inflammatory condition that requires ongoing routine clinical care and treatment to address high disease burden, impaired daily functioning, and reduced health-related quality of life (HRQoL) [1-4]. In this complex disease, interactions between genetic and environmental factors contribute to inflammation and clinical symptoms $[5,6]$. Pruritus due to psoriasis can be intense and is a major determinant of quality of life impairment $[7,8]$. Psoriasis is also accompanied by other distressful symptoms, including skin lesions in highly visible special areas such as the scalp, nails, and palmoplantar areas, as well as other medical and psychological comorbidities [2, 3, 9-11]. Clinical trials assess a treatment's efficacy and safety, but patients who meet stringent inclusion and 
exclusion criteria in clinical trials may not reflect those in real-world clinical practice. Realworld studies can provide valuable insights into the use of psoriasis treatments in diverse types of patients encountered in clinical practice [12]. The use of patient-reported outcomes has gained prominence among healthcare providers as they allow physicians to evaluate treatment effectiveness with regard to the patient's functional status and HRQoL $[13,14]$. Understanding the patient's perspective can also provide insights into reasons for treatment adherence or nonadherence [15].

Treatment effectiveness has been shown to strongly influence treatment satisfaction and may affect patients' treatment preferences. However, studies of patients undergoing treatment for a range of conditions, including psoriasis, show that some patients report high treatment satisfaction despite a lack of treatment effectiveness based on physician- and patient-rated assessments [16-19]. Other aspects of treatment such as safety, route of administration, and compatibility with lifestyle can also affect overall treatment satisfaction [18-20]. Investigating the relationship between patientrelevant benefits and treatment satisfaction can help provide a more holistic approach to assessing the effectiveness of psoriasis treatments [20]. Considering that $52.3 \%$ of patients with psoriasis reported being dissatisfied with their current treatment in the US National Psoriasis Foundations surveys [12], a better understanding of patient-relevant treatment benefits may support efforts to improve treatment satisfaction and effectiveness.

The APPRECIATE study (NCT02740218) was a multinational, retrospective, cross-sectional study of patients with psoriasis who started treatment with apremilast, an oral phosphodiesterase 4 inhibitor, in real-world clinical practice [21]. Apremilast is approved in Europe for the treatment of adult patients with moderate to severe chronic plaque psoriasis who failed to respond to, have a contraindication to, or are intolerant of other systemic therapy, including cyclosporine, methotrexate, or psoralen and ultraviolet A light (PUVA). In APPRECIATE, patients demonstrated notable mean improvements on assessments of disease severity (i.e.,
Psoriasis Area and Severity Index (PASI), psoriasis-involved body surface area (BSA) and HRQoL (i.e., Dermatology Life Quality Index $(\mathrm{DLQI}))$ at $6( \pm 1)$ months after apremilast initiation [21]. The majority of patients in APPRECIATE also achieved the minimum patient-relevant benefit on the Patient Benefit Index (PBI; global PBI score $\geq 1$ ) with ongoing apremilast treatment for $6( \pm 1)$ months [21]. The PBI is a validated patient-reported outcomes measure for skin diseases that evaluates the benefits of treatment with respect to treatment-related patient needs $[22,23]$. The PBI was developed to enable assessment of patient-relevant benefits, taking into account each patient's individual treatment preferences and goals [22]. Here, we further describe patient-relevant needs and benefits in all patients and in subgroups based on clinically relevant patient characteristics. In addition, we analyze the association between patient-relevant benefits and patients' treatment satisfaction.

\section{METHODS}

\section{Participants}

Details of the patient population and study design have been published previously [21]. Briefly, the study enrolled patients who were $\geq 18$ years of age at the time of signing the informed consent form and who had physiciandiagnosed chronic plaque psoriasis [21]. Patients who started apremilast treatment in line with the summary of product characteristics indication during the previous $6( \pm 1)$ months could be enrolled in the study whether or not they had completed 6 months of apremilast treatment. The study excluded patients who were currently participating in another clinical trial (including apremilast clinical trials).

\section{Study Design and Data Collection}

APPRECIATE was a multinational, retrospective, observational, cross-sectional study of patients with psoriasis treated with apremilast in real- 


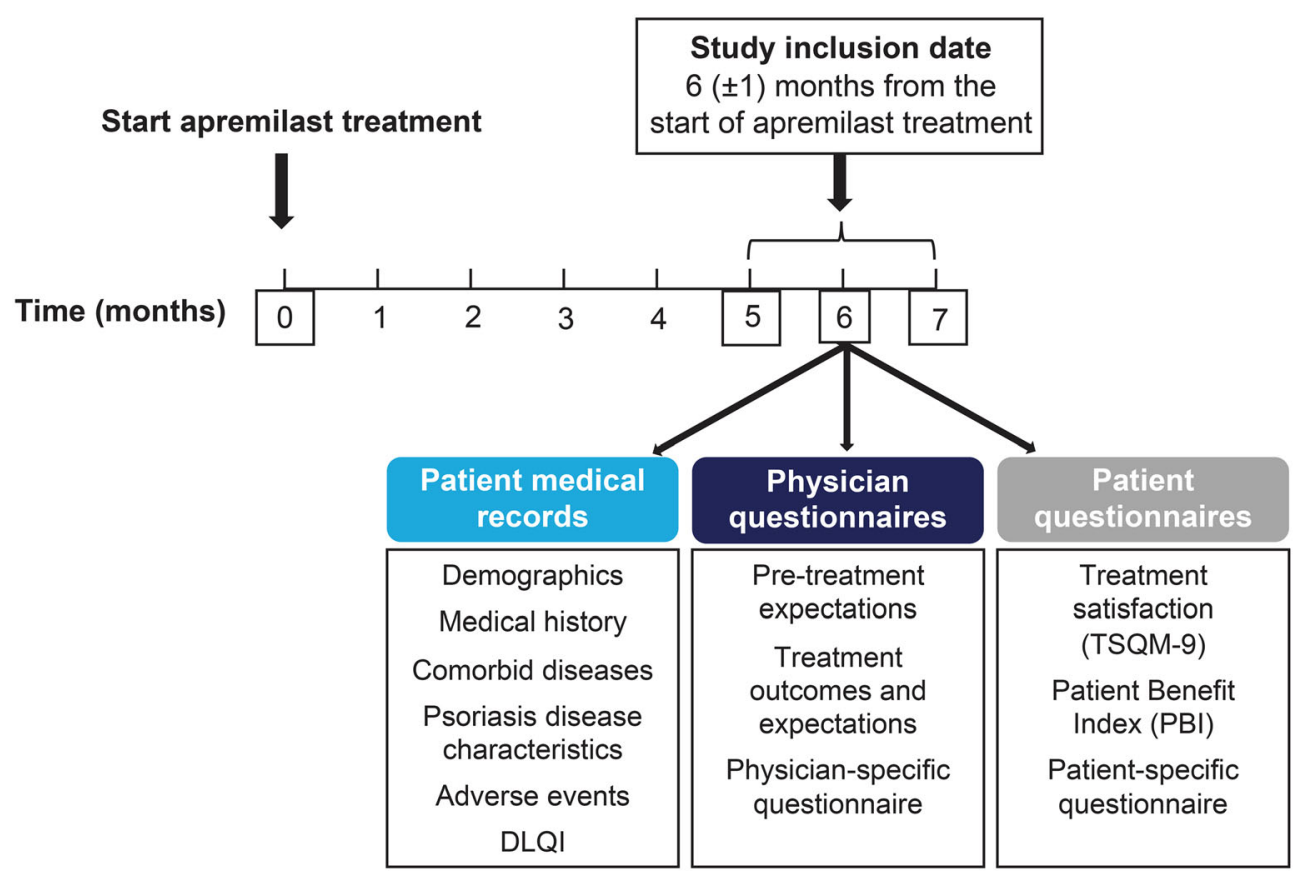

Fig. 1 Study design. Reproduced with permission from Augustin et al. Characteristics and outcomes of patients treated with apremilast in the real world: results from the APPRECIATE study. J Eur Acad Dermatol Venereol.

world clinical settings in Europe (Fig. 1). This analysis was conducted using data from 87 study sites in six countries in Europe (Austria, Germany, Ireland, Sweden, Switzerland, and the UK). Data collection took place between May 2016 and July 2018. The study was approved by the relevant ethics committees and was conducted in accordance with the principles of the Declaration of Helsinki [21]. Written informed consent was obtained from patients before study participation.

At the time of study inclusion (i.e., $6( \pm 1)$ months after initiating apremilast treatment), patient demographics, medical history, comorbid conditions, psoriasis disease characteristics, and adverse events were obtained retrospectively from medical records. All assessments were performed during routine clinical visits. Physicians and patients completed study-specific questionnaires designed to assess patient needs and treatment goals. Patients completed the PBI for skin diseases (PBI-S or PBI) and the
2021;35(1):123-134. Copyright 2020 John Wiley \& Sons, Inc. DLQI Dermatology Life Quality Index, TSQM-9 9-item Treatment Satisfaction Questionnaire for Medication

nine-item Treatment Satisfaction Questionnaire for Medication (TSQM-9).

The PBI weighs treatment benefits in various domains based on their relative importance, as defined by the individual patients' needs $[22,23]$. This measure consists of a patient needs questionnaire (PNQ) and patient benefit questionnaire $(\mathrm{PBQ})$, both of which include the same 25 items (Supplementary Table 1) [21, 23]. The PNQ evaluates the relevance of the 25 items as treatment goals for the individual patient [22]. The PBQ measures the extent to which the current therapy contributed to achieving each treatment goal [22]. The range of possible scores on the PNQ and PBQ is 0 (no importance/benefit) to 4 (maximum importance/benefit) [22]. The PNQ and PBQ are used to calculate the global PBI score, which is calculated as the mean of all PBQ items weighted by the relative importance of corresponding PNQ items for each patient [22]. A global PBI score $\geq 1$ is considered the minimum patient-relevant benefit [23]. High benefit was defined as a global PBI 
score $\geq 3$ [21]. Although the PNQ and PBQ are typically completed before and after treatment [22], both the PNQ and PBQ were completed by the patient at study inclusion (i.e., $6( \pm 1)$ months after apremilast initiation) owing to the retrospective study design of APPRECIATE.

The TSQM-9 is a validated nine-item instrument that includes an effectiveness scale, a convenience scale, and a global satisfaction scale. Scores on each scale range from 0 to 100 , with higher scores indicating greater treatment satisfaction [24].

\section{Statistical Analyses}

All analyses were descriptive for all endpoints and used pooled data from the six European countries participating in APPRECIATE. Data are reported as observed without imputations for missing values (i.e., patients with missing data were excluded). Subgroup analyses of global PBI scores by age group ( $<35$ years, 35-65 years, $>65$ years), sex (male, female), psoriasis in special areas (nail, scalp, palmoplantar, or none), and number of prior systemic treatments or phototherapies $(0,1,2$, or $>2)$ were conducted. Additional subgroup analyses evaluated global PBI scores by type of prior treatment (i.e., patients were allocated to one category in the following hierarchical ranking order: no prior treatment, phototherapy (but no other treatment), conventional systemic (may have had phototherapy but no other systemics), biologic (may have had phototherapy and/or conventional systemics)); by previous conventional systemic treatment (i.e., patients who received prior methotrexate but not fumarates, patients who received prior fumarates but not methotrexate, or patients who previously received both methotrexate and fumarates); by presence or absence of symptoms of psoriasis or psoriasis in special areas (i.e., pruritus or scalp, nail, or palmoplantar involvement); by age at psoriasis diagnosis ( $\leq 40$ years, $>40$ years); and by presence or absence of psoriatic arthritis (PsA). The top needs (responses of 'quite' and 'very' on the PNQ) and responses indicating at least a moderate benefit for the top needs after apremilast treatment (responses of 'moderately', 'quite', or 'very' on the PBQ) were evaluated in patients continuing apremilast treatment at $6( \pm 1)$ months and in a post hoc subgroup analysis of patients with and without PsA. Preplanned correlation analyses using Pearson's correlation coefficients were performed to evaluate the association between the global PBI score and TSQM-9 global satisfaction score, TSQM-9 effectiveness score, and TSQM-9 convenience score.

\section{RESULTS}

\section{Patients}

Of the 605 patients who were contacted about participating, 484 agreed to participate and enrolled in the study [21]; four patients were later determined to be ineligible and were excluded from analyses (Fig. 2). A total of $124 / 480(25.8 \%)$ patients in the full analysis population had PsA, 64 (51.6\%) of whom had a rheumatologist-confirmed diagnosis. A total of $347 / 480(72.3 \%)$ patients were continuing treatment with apremilast at the 6-month assessment (Fig. 2). Among patients continuing apremilast with a PBI score available for analysis, 36/330 (10.9\%) patients had no prior treatment, 65/330 (19.7\%) had prior phototherapy, $184 / 330(55.8 \%)$ had prior conventional systemic treatment, and 44/330 (13.3\%) had prior biologic treatment, as assigned to a single category by hierarchical order (i.e., in the order presented above; Fig. 2). One patient (0.2\%) received other prior treatment.

Demographics and disease characteristics for the 480 patients in the study population have been previously reported [21]. Briefly, mean age was 51.3 (standard deviation $(\mathrm{SD})=15.2$ ) years, and $53.8 \%(n=258)$ of patients were male. The mean time since psoriasis diagnosis was 18.6 $(\mathrm{SD}=14.3)$ years. At apremilast initiation, mean psoriasis-involved BSA was $25.4 \%$ (SD $=23.5 \%, n=141)$, mean PASI score was 12.5 $(\mathrm{SD}=8.4, n=350)$, and mean DLQI score was $13.4(\mathrm{SD}=7.5, n=205)$. 


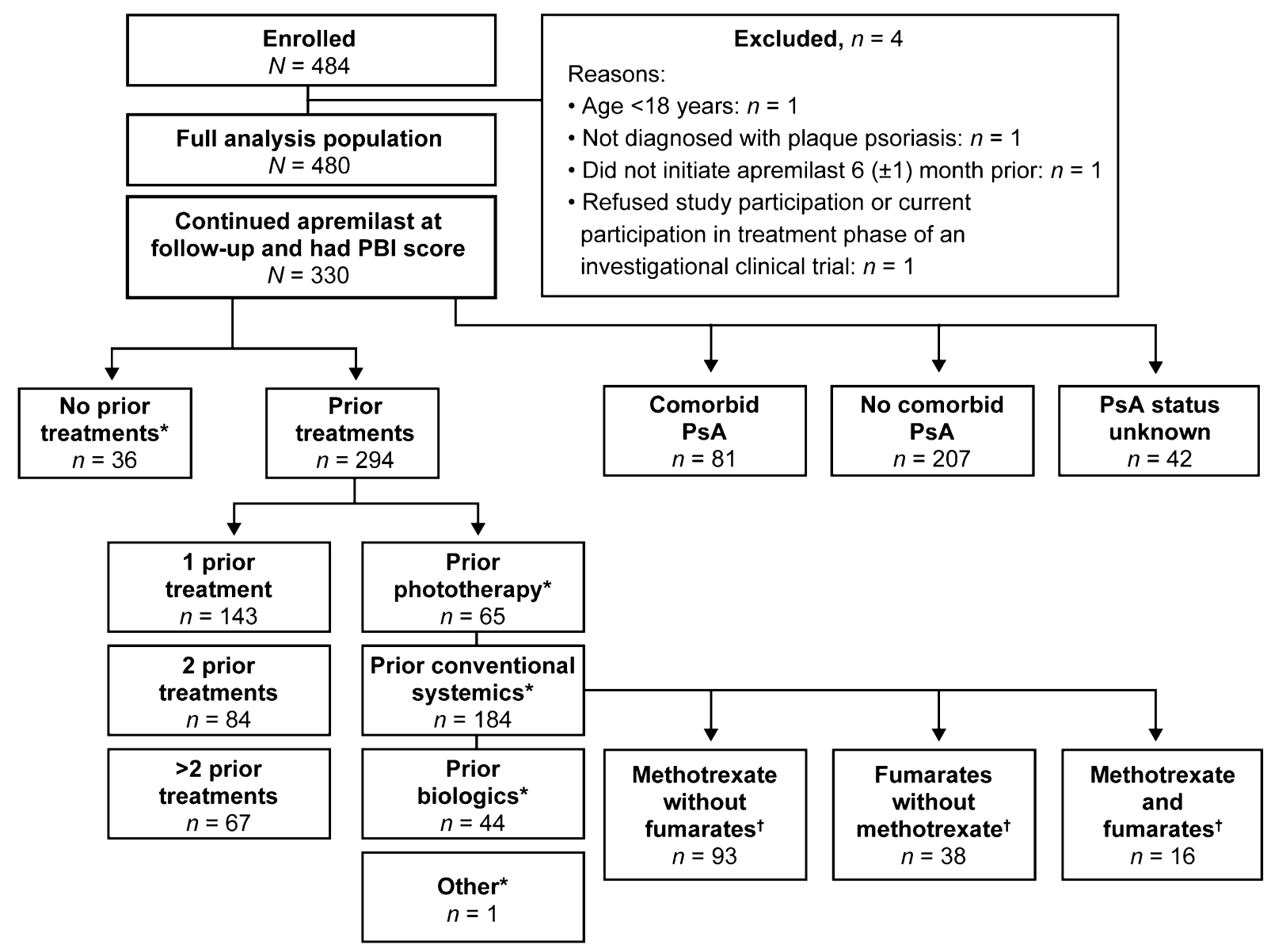

Fig. 2 Patient disposition. Note that number and type of prior treatments are not mutually exclusive. ${ }^{*}$ Each patient was allocated to only one prior treatment category (in the following hierarchical ranking order: no prior treatment, prior phototherapy (but no other treatment), prior conventional systemic (may have had phototherapy but no other systemics), and prior biologic (may have had

\section{PBI Analyses}

There were $451 / 480$ patients (94.0\%) with a PBI score available for analysis. The mean global PBI score was $2.4(\mathrm{SD}=1.4)$ among all patients and $2.8 \quad(\mathrm{SD}=1.2) \quad$ among patients continuing apremilast at $6( \pm 1)$ months (Table 1) [21]. In patients continuing apremilast treatment, $52.4 \%(182 / 347)$ reported a high treatment benefit (global PBI score $\geq 3$ ). Among patients continuing apremilast treatment with a PBI score available for analysis $(n=330)$, types of prior treatments included: biologics $(n=44)$, phototherapy and/or conventional systemics)) even if more than one prior psoriasis therapy had been documented. One patient received other treatment. ${ }^{\dagger}$ Among patients on apremilast treatment at follow-up who were allocated to prior conventional systemic treatment based on the hierarchy. Patients may have received other conventional systemics (e.g., retinoids)

including adalimumab $(22 / 44,50.0 \%)$, etanercept $(15 / 44,34.1 \%)$, ustekinumab (9/44, $20.5 \%)$, secukinumab $(9 / 44,20.5 \%)$, infliximab $(6 / 44,13.6 \%)$, efalizumab $(3 / 44,6.8 \%)$, ixekizumab $(2 / 44,4.5 \%)$, tildrakizumab (1/44, $2.3 \%)$, and certolizumab (1/44, 2.3\%); conventional systemics $(n=184), \quad$ including methotrexate $(109 / 184,59.2 \%)$, fumaric acid $(54 / 184,29.3 \%)$, acitretin $(34 / 184,18.5 \%)$, cyclosporine $(23 / 184,12.5 \%)$, retinoids (14/ $184,7.6 \%)$, glucocorticoids (9/184, 4.9\%), leflunomide $(2 / 184,1.1 \%)$, and steroids $(1 / 184$, $0.5 \%)$; and phototherapy $(n=65)$, including 
Table 1 Global PBI scores for patients on apremilast treatment at follow-up

\begin{tabular}{lcc}
\hline & $n$ & Mean (SD) \\
\hline Global PBI score & 330 & $2.8(1.2)$ \\
$\begin{array}{l}\text { Global PBI score by number of prior systemic treatments } \\
\text { or phototherapies }\end{array}$ & & \\
0 & 36 & $3.0(1.1)$ \\
1 & 143 & $2.8(1.1)$ \\
2 & 84 & $2.8(1.2)$ \\
$>2$ & 67 & $2.5(1.3)$
\end{tabular}

Global PBI score by type of prior treatment*

\begin{tabular}{lll} 
None & 36 & $3.0(1.1)$ \\
Phototherapy & 65 & $3.0(1.0)$ \\
Conventional systemics & 184 & $2.8(1.2)$ \\
Biologics & 44 & $2.4(1.3)$ \\
Global PBI score by prior treatment & & \\
Methotrexate without fumarates $^{\dagger}$ & 93 & $2.7(1.2)$ \\
Fumarates without methotrexate $^{\dagger}$ & 38 & $2.8(1.2)$ \\
Both methotrexate and fumarates $^{\dagger}$ & 16 & $2.6(1.2)$ \\
\hline
\end{tabular}

Data shown are based on patients who continued apremilast at $6( \pm 1)$ months' follow-up and answered the PBI $(n=330)$. Possible scores range from 0 (no benefit) to 4 (maximal benefit)

PBI Patient Benefit Index, SD standard deviation

*Each patient was allocated to only one prior treatment category (by hierarchical order as displayed) even if more than one prior psoriasis therapy had been documented. One patient received other treatment

${ }^{\dagger}$ Patients may have received other conventional systemics (e.g., retinoids)

narrow band UVB (30/65, 46.2\%), UVA/UVB $(n=22 / 65,33.8 \%)$, PUVA $(13 / 65,20.0 \%)$, and one $(1.5 \%)$ unknown.

The top ten patient treatment needs, as identified in the PNQ, are presented in Fig. 3 for patients with non-missing data. More than 90\% of patients responded that 'regain control of the disease', 'have confidence in the therapy', 'get better skin quickly', and 'be healed of all skin defects' were quite or very important needs.
More than $48 \%$ of patients reported a benefit (answers of quite or very) on the PBQ for all of the top ten treatment needs; seven of the top ten benefits overall corresponded to the top ten needs identified in the PNQ (Fig. 4).

Similar mean global PBI scores were observed in patients without $(2.3(\mathrm{SD}=1.5))$ versus those with $(2.4(\mathrm{SD}=1.4)) \geq 1$ symptom of psoriasis or psoriasis in special areas (i.e., pruritus or scalp, nail, or palmoplantar involvement). In a subgroup analysis of global PBI scores based on number of prior systemic treatments, mean global PBI scores generally decreased as number of prior systemic treatments for psoriasis increased, and the lowest mean PBI scores were observed for patients who had prior biologic treatment (Table 1). In patients with prior conventional systemic treatment, mean PBI scores were generally similar among patients whose prior treatment included methotrexate without fumarates, fumarates without methotrexate, or both methotrexate and fumarates (Table 1). The proportion of patients reporting a high benefit (global PBI score $\geq 3$ ) was greater in patients who had received fewer prior treatments versus those with more prior treatments; in patients who had received prior treatment with phototherapy versus conventional systemics and biologics; and in patients who had prior treatment with fumarates but not methotrexate versus prior methotrexate but not fumarates or prior treatment with both fumarates and methotrexate (Fig. 5).

Mean global PBI scores at follow-up were similar in patients with versus without comorbid PsA (2.8 ( $\mathrm{SD}=1.0)$ versus $2.8(\mathrm{SD}=1.2)$, respectively). Patients with and without comorbid PsA shared eight of ten top treatment needs based on the PNQ. The two treatment goals of 'to have fewer side effects' and 'to be free of pain' were identified as high needs only in patients with comorbid PsA (83.9\% (99/118) and $82.2 \%$ (97/118), respectively), and the two treatment goals of 'need less time for daily treatment' and 'be able to engage in normal leisure activities' were identified as high needs only in patients without comorbid PsA (81.2\% $(233 / 287)$ and $77.9 \%(225 / 289)$, respectively; Fig. S1). Most patients with and without PsA 


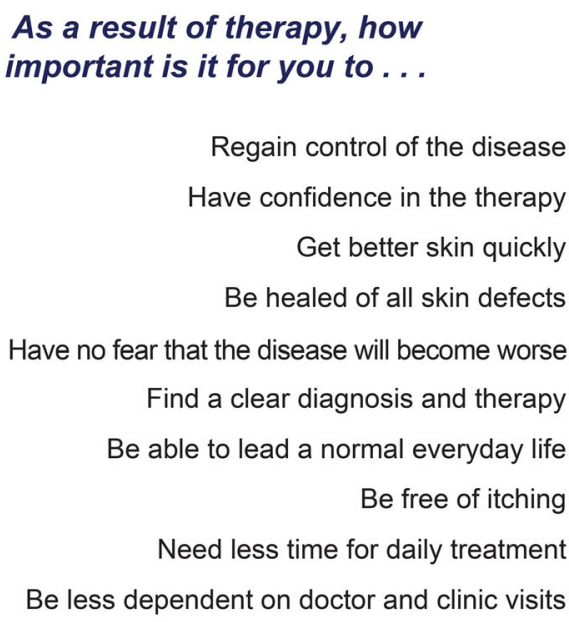

Fig. 3 Top ten needs of patients, as identified in the PNQ. FAS, $N=480$; number of patients with data available may vary. Possible answers include $0=$ not at all/does not apply to me, $1=$ somewhat, $2=$ moderately, $3=$ quite, and $4=$ very. The top ten needs of patients

reported a high benefit on all of their ten top treatment needs (Fig. S1).

Similar mean global PBI scores were observed in subgroups of patients based on age $(<35$ years: $2.3(\mathrm{SD}=1.4)$; 35-65 years: 2.4 (SD $=1.4) ;>65$ years: $2.5(\mathrm{SD}=1.3)$ ), sex (male: 2.3 $(\mathrm{SD}=1.4)$; female: $2.4(\mathrm{SD}=1.4))$, and age at psoriasis diagnosis ( $\leq 40$ years: 2.3 (SD $=1.4) ;>40$ years: $2.5(\mathrm{SD}=1.3))$. The proportions of patients reporting a high benefit of apremilast treatment (global PBI score $\geq 3$ ) were greater in older patients ( $>65$ years: $47.1 \%$ $(49 / 104))$ versus younger patients $(<35$ years: 40.5\% (32/79); 35-65 years: 40.7\% (121/297)) and similar in subgroups based on sex (male: $42.2 \%$ (109/258); female: $41.9 \%(93 / 222)$ ) and age at psoriasis diagnosis ( $\leq 40$ years: $41.5 \%$ $(136 / 328)$; $>40$ years: $43.5 \%(64 / 147))$.

\section{Association Between Global PBI Scores and Ratings of Apremilast Treatment Satisfaction and Effectiveness}

Mean TSQM subscale scores were 59.8 (SD $=28.9 ; n=455)$ for the effectiveness subscale, $82.4(\mathrm{SD}=18.0 ; n=462)$ for the convenience
High Agreement

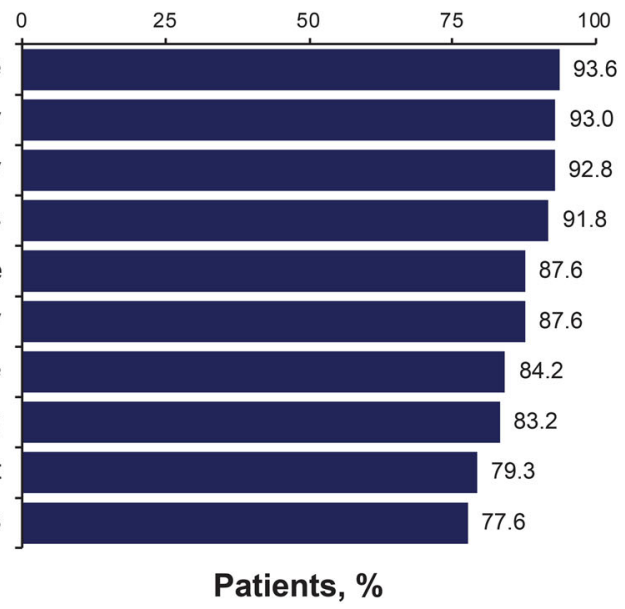

(answered 'quite' or 'very' by the most patients) out of 25 needs on the PNQ are presented. Positive response includes those answering 'quite' or 'very'. Percentage calculation excludes missing answers. $F A S$ full analysis set, $P N Q$ Patient Needs Questionnaire

subscale, and 58.2 ( $\mathrm{SD}=31.2 ; n=462$ ) for the global satisfaction subscale. Strong positive associations were observed between the global PBI score and the global satisfaction and effectiveness TSQM-9 subscale scores, respectively (Fig. 6). A weak positive correlation was found between global PBI score and the convenience subscale of the TSQM-9 (Fig. 6).

\section{DISCUSSION}

Patient-reported outcome measures can provide valuable assessments of treatment benefits that are important for patients' lives [13, 22]. Because some factors that contribute to overall disease burden, such as pruritus and psoriasis in special areas, are not fully captured by traditional clinical measures of disease severity, patient-reported outcome measures can help physicians to obtain a broader evaluation of treatment effectiveness $[13,14,25,26]$. The PBI is an assessment of patient-relevant treatment benefits validated for use with psoriasis patients $[22,23]$. In the APPRECIATE study, apremilast demonstrated improvements in disease severity measures at $6( \pm 1)$ months after apremilast 
(a) Apremilast has helped me to...

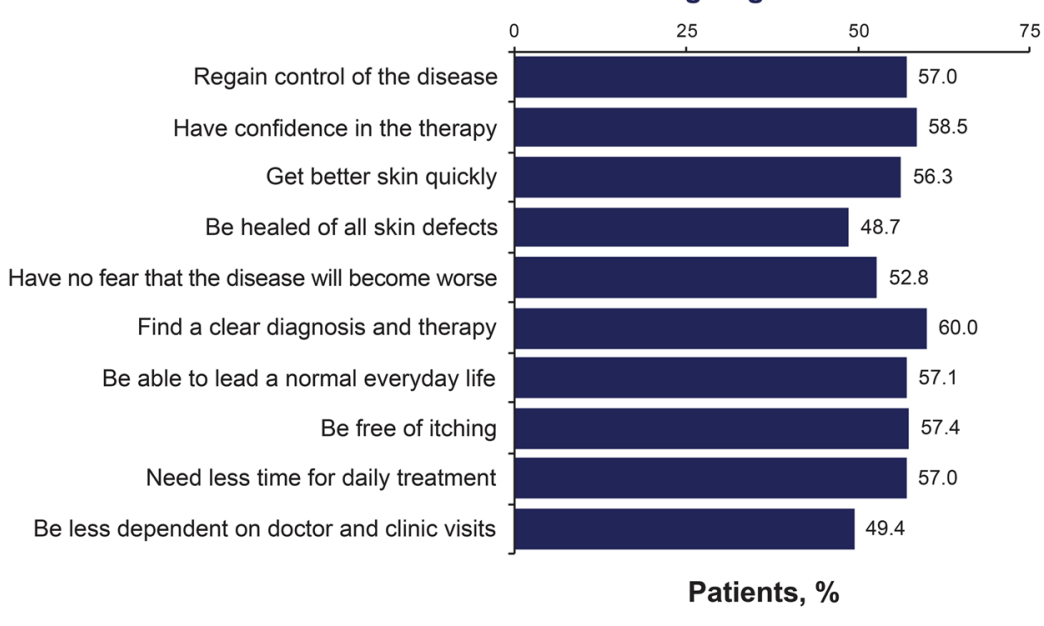

(b) Apremilast has helped me to...

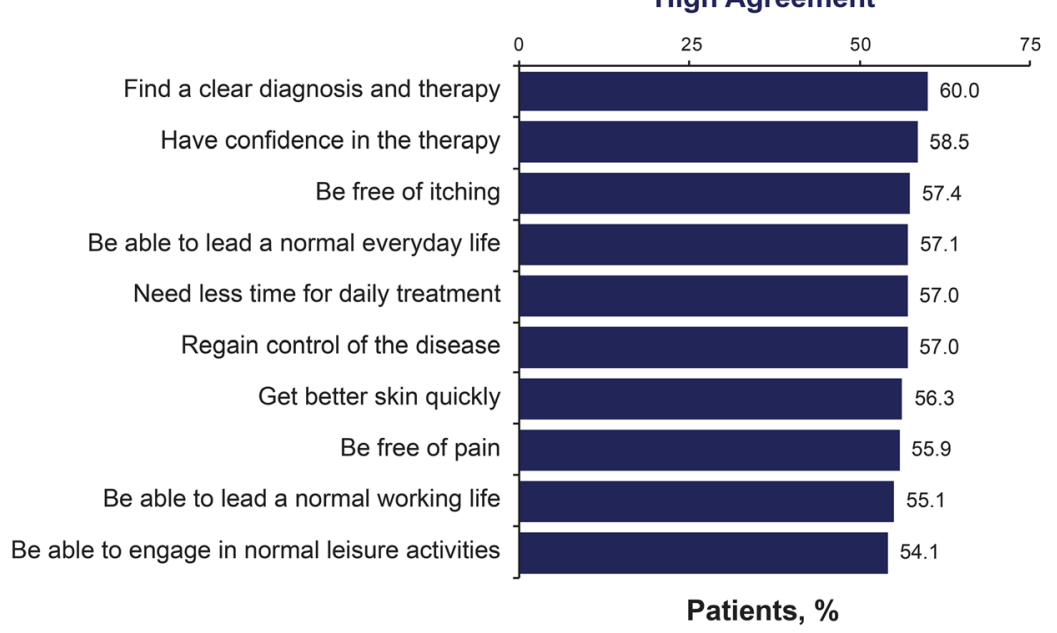

Fig. 4 A Benefit response for top ten needs on the PNQ and $\mathbf{B}$ top ten benefit responses overall, as identified in the PBQ. FAS, $N=480$; number of patients with data available may vary. Percentage calculation excluded missing and 'does not apply to me' answers. Possible answers

initiation [21]. Results from the current analysis of patient-reported outcomes from APPRECIATE suggest that patients continuing apremilast treatment at $6( \pm 1)$ months experienced treatment benefits, particularly patients with no prior treatments and those without prior conventional systemic or biologic treatment. The proportion of patients who reported a high benefit (defined as global PBI score $\geq 3$ ) with 6 $( \pm 1)$ months of apremilast treatment was include $0=$ not at all $/$ does not apply to me, $1=$ somewhat, $2=$ moderately, $3=$ quite, and $4=$ very. The benefits are defined as positive responses including answers of 'quite' or 'very'. FAS full analysis set, $P B Q$ Patient Benefit Questionnaire

highest among patients who had been previously treated with phototherapy or who had not been previously treated with any other systemic psoriasis treatment. Our findings suggest that receiving apremilast treatment earlier rather than later in treatment management is consistent with greater improvements in patient-relevant treatment outcomes. This might be due to increased benefit of apremilast in this patient group. 


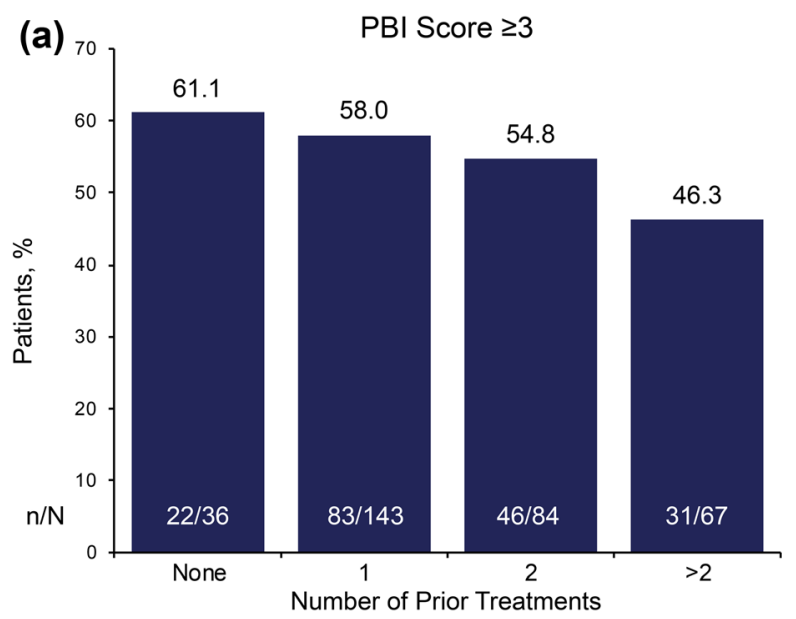

(b)

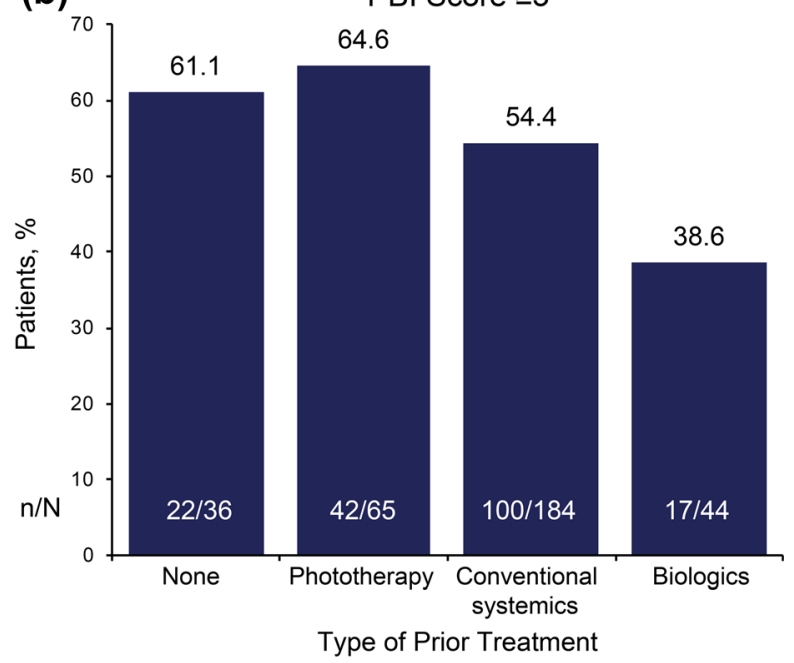

(c)

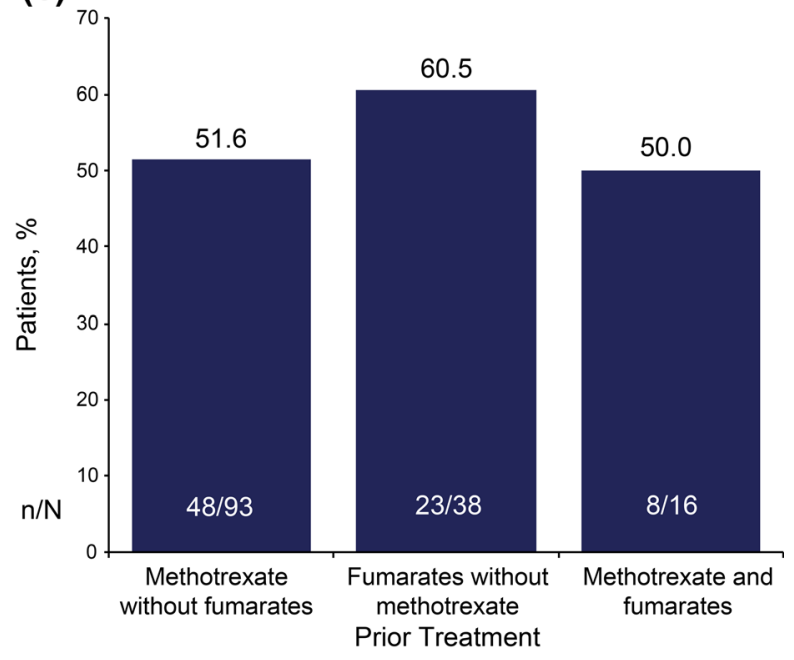

4 Fig. 5 Patients achieving a high benefit (PBI score $\geq 3$ ) on apremilast treatment at follow-up by $\mathbf{A}$ number of prior treatments; $\mathbf{B}$ type of prior treatment; and $\mathbf{C}$ prior treatment: methotrexate without fumarates, fumarates without methotrexate, or both methotrexate and fumarates. ${ }^{*}$ Excludes missing data. $n / N$ number of patients who reported $\mathrm{PBI} \geq 3 /$ number of patients in prior treatment category. Data shown are based on patients who continued apremilast at follow-up and answered the PBI $(n=330)$. *Patients may have received other conventional systemics (e.g., retinoids). PBI Patient Benefit Index

Based on patients' responses to the PBI, the treatment needs of psoriasis patients in APPRECIATE extended beyond skin improvement and encompassed other physical, psychological, and other patient-relevant benefits associated with apremilast treatment. Many of the top ten needs identified in the APPRECIATE study were consistent with the top needs identified by psoriasis patients in a validation study of the PBI [22], as well as analyses of PBI data from large psoriasis registries in Europe (the German PsoBest registry and the national psoriasis registry of Switzerland) [27, 28]. Our findings on the PNQ and PBQ items in the APPRECIATE study revealed that most patients continuing apremilast treatment for $6( \pm 1)$ months $(72.3 \%$ of enrolled patients) reported treatment benefits on the top patient-reported key treatment needs. APPRECIATE patients with comorbid PsA shared most of the same treatment needs and had similar benefit responses as patients without PsA and the overall study population; however, being free of pain and having fewer side effects were top treatment needs in patients with PsA but not in patients without PsA.

In the APPRECIATE study, patient ratings of global satisfaction and treatment effectiveness of apremilast were strongly correlated with the global PBI score, thus confirming that the PBI measure can provide insights into psoriasis treatment effectiveness that are relevant to patients and physicians. In addition, a small group of patients had a high global PBI score but low satisfaction with treatment effectiveness, suggesting that patients also consider 

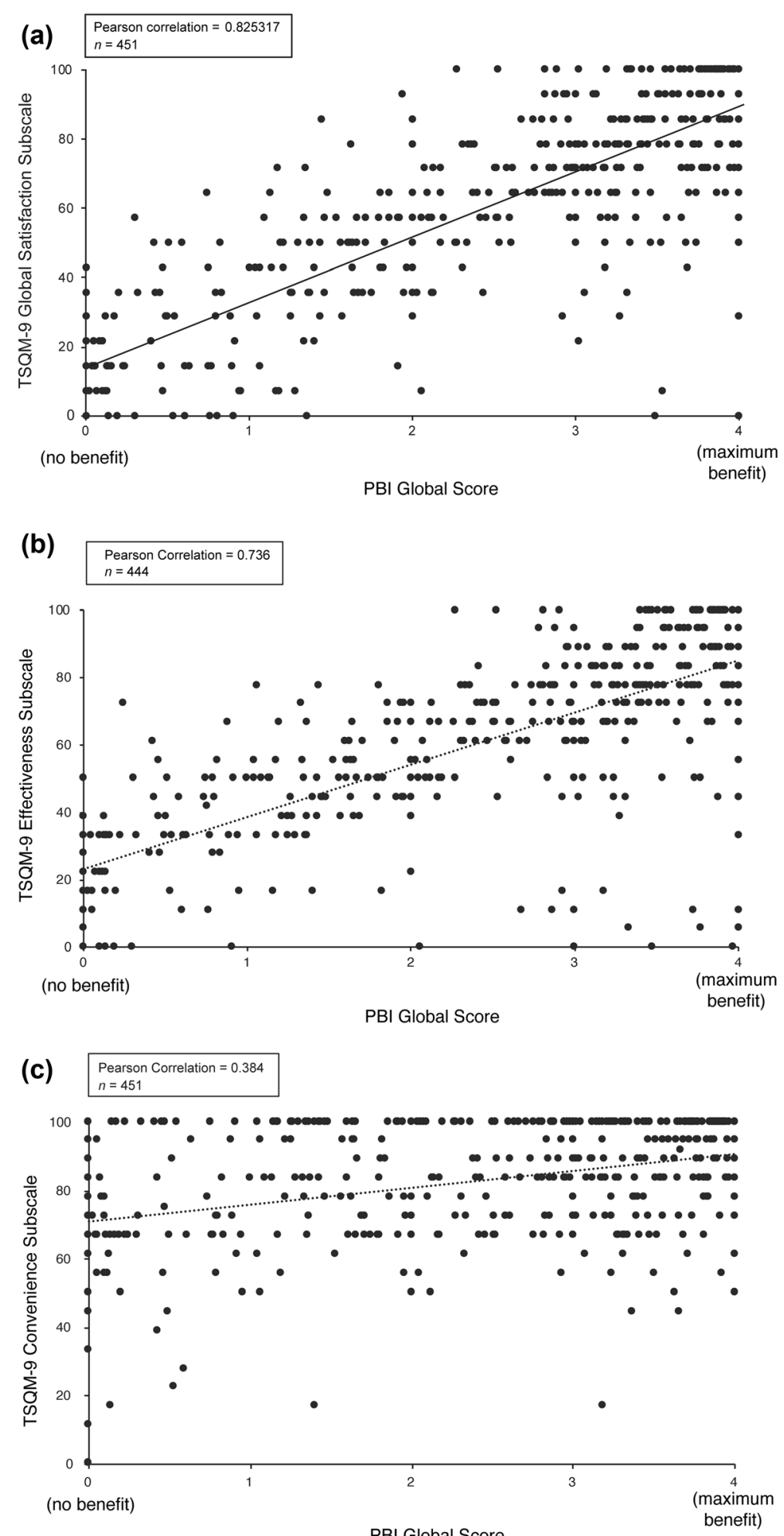

Fig. 6 Correlation analysis of global PBI score versus A TSQM-9 global satisfaction score; B TSQM-9 effectiveness score; and C TSQM-9 convenience score. PBI
Patient Benefit Index, TSQM-9 nine-item Treatment Satisfaction Questionnaire for Medication 
factors other than treatment effectiveness when assessing their overall satisfaction with apremilast treatment. In support of this concept, a systematic review that evaluated treatment preferences and satisfaction among psoriasis patients found that some patients rated attributes associated with the treatment process (such as access or mode of delivery) as more important than efficacy outcomes [20]. In APPRECIATE, patient ratings of the convenience of apremilast treatment only weakly correlated with patient assessments of apremilast benefits, and this weak correlation may reflect that ratings of treatment benefit may not be based on treatment convenience.

Some limitations of this study include those inherent to retrospective analyses such as recall bias and data that are missing or not recorded based on chart review. Analyses including patients who continued treatment may not be representative of all patients who started or stopped treatment; the benefit of apremilast may differ in the overall population of study patients who started apremilast treatment, and data have shown that patients in APPRECIATE who discontinued apremilast treatment had lower global PBI scores and TSQM-9 subscale scores than those who continued treatment [21]. Also, confounding was possible in the prior treatment subgroups because patients who had greater numbers of prior treatments may have been more likely to have had prior biologic treatment. As the prior treatment subgroups were not randomized, some characteristics may have been unbalanced between the subgroups. The number of prior treatments may have been related to disease severity or may have influenced the patient's ability to assess treatment benefits, and these considerations may limit the interpretation of study findings. Also, findings should be interpreted with caution owing to the small sample sizes in some of the subgroups. In addition, because there were no predefined criteria to screen and enroll patients with comorbid PsA, patients with comorbid PsA in this study may not be representative of the general population of patients with psoriasis and comorbid PsA.

\section{CONCLUSION}

Continuing apremilast treatment for $6( \pm 1)$ months was associated with patient-relevant treatment benefits in diverse patient subgroups, including in patients with and without prior conventional systemic or biologic treatments, symptoms such as pruritus, psoriasis in special areas, and comorbid PsA. Results from the APPRECIATE study suggest that patients may benefit from apremilast treatment earlier rather than later in treatment management. This analysis also underscores the value of evaluating parameters that are relevant to patients' lives using a measure such as the PBI when assessing treatment effectiveness in clinical practice.

\section{ACKNOWLEDGEMENTS}

The authors would like to thank all study investigators, site personnel, and patients who participated in the study. The authors thank Lillian Mellars for her contribution.

Funding. This study and the journal's Rapid Service fees were funded by Amgen Inc. Amgen acquired the worldwide rights to Otezla ${ }^{\circledR}$ (apremilast) from Celgene on November 21, 2019.

Medical Writing and Editorial Assistance. Writing support was funded by Amgen Inc. and provided by Mandy Suggitt of Amgen Inc. and Amy Shaberman, PhD, of Peloton Advantage, LLC, an OPEN Health company.

Authorship. All named authors meet the International Committee of Medical Journal Editors (ICMJE) criteria for authorship for this article, take responsibility for the integrity of the work as a whole, and have given their approval for this version to be published.

Author Contributions. TMK: Analysis planning, analysis and interpretation of data, drafting the manuscript, and review of the final draft. C Blome: Analysis and interpretation of data, drafting the manuscript, and review of the 
final draft. CEK: Patient data collection/acquisition of data; analysis and interpretation of data, drafting the manuscript, and review of the final draft. CC: Patient data collection/acquisition of data; analysis and interpretation of data, drafting the manuscript, and review of the final draft. PGS: Patient data collection/acquisition of data; analysis and interpretation of data, drafting the manuscript, and review of the final draft. MS: Patient data collection/acquisition of data; analysis and interpretation of data, drafting the manuscript, and review of the final draft. KE: Patient data collection/acquisition of data; analysis and interpretation of data, drafting the manuscript, and review of the final draft. MAR: Patient data collection/acquisition of data; analysis and interpretation of data, drafting the manuscript, and review of the final draft. C Bundy: Patient data collection/acquisition of data; analysis and interpretation of data, drafting the manuscript, and review of the final draft. MC: Conception and design of study; analysis and interpretation of data, drafting the manuscript, and review of the final draft. CEMG: Patient data collection/acquisition of data; analysis and interpretation of data, drafting the manuscript, and review of the final draft. MA: Patient data collection/acquisition of data; analysis and interpretation of data, drafting the manuscript, and review of the final draft.

Prior Presentations. Griffiths CEM, et al. Real-World Experience With Apremilast: Analysis of Patients From the APPRECIATE Study With Specific Manifestations of Psoriasis. Presented at: the 50th Deutsche Dermatologische Gesellschaft; May 1-4, 2019; Berlin, Germany. Augustin M, et al. Real-World Experience With Apremilast: Influence of Prior Therapy on Patient Benefit in Patients With Psoriasis From the APPRECIATE Study. Presented at: the 50th Deutsche Dermatologische Gesellschaft; May 1-4, 2019; Berlin, Germany. Kleyn CE, et al. Apremilast Real-World Treatment Outcomes in Psoriasis Patients With Comorbid Psoriatic Arthritis: An Analysis of the APPRECIATE Study. Presented at: the 28th European Academy of Dermatology and Venereology (EADV) Congress; October 9-13, 2020; Madrid, Spain.
Disclosures. Toni Maria Klein: No conflicts of interest. Christine Blome: Celgene Corporation, Janssen-Cilag, Kreussler, Lilly, Mapi Group, medi, Stiefel Laboratories, The EuroQol Group, and Urgo-speaker honoraria, research grants, awards, and/or travel expenses. C. Elise Kleyn: AbbVie, Almirall, Amgen Inc., Celgene Corporation, Janssen, Lilly, Novartis, Pfizer, and UCB Pharma-honoraria, consultancy, and/or research grants. Curdin Conrad: AbbVie, Actelion, Almirall, Amgen Inc., Bristol Myers Squibb, Celgene Corporation, Galderma, Incyte, Janssen, LEO Pharma, Lilly, MSD, Novartis, Pfizer, Samsung, and UCB-scientific advisor, clinical study investigator, and/or paid speaker. Paul G. Sator: Abbott, AbbVie, Actelion, ALK, Amgen Inc., Almirall, Celgene Corporation, Galderma, Gilead, Janssen, LEO Pharma, Lilly, Maruho, MSD, Novartis, Pfizer, and UCB-research grants, speaker and/or consulting honoraria, and/or travel refunds. Mona Ståhle: AbbVie, Amgen Inc., Celgene Corporation, Eli Lilly, Janssen-Cilag, LEO Pharma, Novartis, Pfizer, and UCB-speaker and advisory board honoraria. Kilian Eyerich: AbbVie, Almirall, Boehringer Ingelheim, Bristol Myers Squibb, Celgene Corporation, Lilly, Janssen, LEO Pharma, Novartis, Sanofi, and UCB-speaker and/or consulting honoraria. Marc Alexander Radtke: AbbVie, Almirall, Celgene Corporation, Janssen, LEO Pharma, Lilly, MSD, Novartis, and Pfizer-speaker and/or clinical trial investigator. Christine Bundy: AbbVie, Almirall, Amgen Inc., Beiersdorf, Janssen, LEO Pharma, Novartis, and Pfizer-research support, speaker/consultancy fees. Myriam Cordey: Amgen Inc.-employment. Christopher E.M. Griffiths: AbbVie, Almirall, Amgen Inc., Boehringer Ingelheim, Bristol Myers Squibb, Celgene Corporation, Janssen, Lilly, Novartis, Sandoz, and UCB Pharma-honoraria and/or research grants; supported in part by the NIHR Manchester Biomedical Centre. Matthias Augustin: AbbVie, Almirall, Amgen Inc., Biogen, Boehringer Ingelheim, Celgene Corporation, Centocor, Eli Lilly, GSK, Hexal, Janssen, LEO Pharma, Medac, Merck, MSD, Mundipharma, Novartis, Pfizer, Sandoz, UCB Pharma, and XenoPort-consulting fees and/or research grants. 
Compliance With Ethics Guidelines. The study was approved by the relevant ethics committees and was conducted in accordance with the principles of the Declaration of Helsinki [21]. Written informed consent was obtained from patients before study participation.

Data Availability. Data sharing is not applicable to this article as no datasets were generated or analyzed during the current study.

Open Access. This article is licensed under a Creative Commons Attribution-NonCommercial 4.0 International License, which permits any non-commercial use, sharing, adaptation, distribution and reproduction in any medium or format, as long as you give appropriate credit to the original author(s) and the source, provide a link to the Creative Commons licence, and indicate if changes were made. The images or other third party material in this article are included in the article's Creative Commons licence, unless indicated otherwise in a credit line to the material. If material is not included in the article's Creative Commons licence and your intended use is not permitted by statutory regulation or exceeds the permitted use, you will need to obtain permission directly from the copyright holder. To view a copy of this licence, visit http:// creativecommons.org/licenses/by-nc/4.0/.

\section{REFERENCES}

1. Baliwag J, Barnes DH, Johnston A. Cytokines in psoriasis. Cytokine. 2015;73(2):342-50.

2. Langley RG, Krueger GG, Griffiths CE. Psoriasis: epidemiology, clinical features, and quality of life. Ann Rheum Dis. 2005;64(Suppl 2):ii18-23 (discussion ii24-15).

3. Lebwohl MG, Bachelez H, Barker J, et al. Patient perspectives in the management of psoriasis: results from the population-based Multinational Assessment of Psoriasis and Psoriatic Arthritis Survey. J Am Acad Dermatol. 2014;70(5):871-81.

4. Sampogna F, Tabolli S, Abeni D. Living with psoriasis: prevalence of shame, anger, worry, and problems in daily activities and social life. Acta Derm Venereol. 2012;92(3):299-303.

5. Kocic H, Damiani G, Stamenkovic B, et al. Dietary compounds as potential modulators of microRNA expression in psoriasis. Ther Adv Chronic Dis. 2019. https://doi.org/10.1177/2040622319864805.

6. Carvalho AL, Hedrich CM. The molecular pathophysiology of psoriatic arthritis-the complex interplay between genetic predisposition, epigenetics factors, and the microbiome. Front $\mathrm{Mol}$ Biosci. 2021;8:662047.

7. Damiani G, Cazzaniga S, Conic RR, Naldi L. Pruritus characteristics in a large Italian cohort of psoriatic patients. J Eur Acad Dermatol Venereol. 2019;33(7): 1316-24.

8. Mrowietz U, Chouela EN, Mallbris L, et al. Pruritus and quality of life in moderate-to-severe plaque psoriasis: post hoc explorative analysis from the PRISTINE study. J Eur Acad Dermatol Venereol. 2015;29(6):1114-20.

9. Harden JL, Krueger JG, Bowcock AM. The immunogenetics of psoriasis: a comprehensive review. J Autoimmun. 2015;64:66-73.

10. Augustin M, Reich K, Glaeske G, Schaefer I, Radtke M. Co-morbidity and age-related prevalence of psoriasis: analysis of health insurance data in Germany. Acta Derm Venereol. 2010;90(2):147-51.

11. Kleyn CE, Talbot PS, Mehta NN, et al. Psoriasis and mental health workshop report: exploring the links between psychosocial factors, psoriasis, neuroinflammation and cardiovascular disease risk. Acta Derm Venereol. 2020;100(1):adv00020.

12. Armstrong AW, Robertson AD, Wu J, Schupp C, Lebwohl MG. Undertreatment, treatment trends, and treatment dissatisfaction among patients with psoriasis and psoriatic arthritis in the United States: findings from the National Psoriasis Foundation surveys, 2003-2011. JAMA Dermatol. 2013;149(10): 1180-5.

13. Weldring T, Smith SM. Patient-reported outcomes (PROs) and patient-reported outcome measures (PROMs). Health Serv Insights. 2013;6:61-8.

14. Mueller SM, Itin PH, Navarini AA, et al. The relationship between PASI and DLQI with itch, stress, and depression: do we need additional decisionmaking tools in psoriasis? Dermatol Ther. 2020;33(3):e13276.

15. Schaarschmidt ML, Kromer C, Herr R, et al. Patient preferences for biologicals in psoriasis: top priority of safety for cardiovascular patients. PLoS One. 2015;10(12):e0144335. 
16. Schaarschmidt ML, Schmieder A, Umar N, et al. Patient preferences for psoriasis treatments: process characteristics can outweigh outcome attributes. Arch Dermatol. 2011;147(11):1285-94.

17. Sumpton D, Kelly A, Tunnicliffe DJ, et al. Patients' perspectives and experience of psoriasis and psoriatic arthritis: a systematic review and thematic synthesis of qualitative studies. Arthritis Care Res (Hoboken). 2020;72(5):711-22.

18. Schafer I, Hacker J, Rustenbach SJ, Radtke M, Franzke N, Augustin M. Concordance of the Psoriasis Area and Severity Index (PASI) and patient-reported outcomes in psoriasis treatment. Eur J Dermatol. 2010;20(1):62-7.

19. Black N, Varaganum M, Hutchings A. Relationship between patient reported experience (PREMs) and patient reported outcomes (PROMs) in elective surgery. BMJ Qual Saf. 2014;23(7):534-42.

20. Florek AG, Wang CJ, Armstrong AW. Treatment preferences and treatment satisfaction among psoriasis patients: a systematic review. Arch Dermatol Res. 2018;310(4):271-319.

21. Augustin M, Kleyn CE, Conrad C, et al. Characteristics and outcomes of patients treated with apremilast in the real world: results from the APPRECIATE study. J Eur Acad Dermatol Venereol. 2021;35(1):123-34.

22. Augustin M, Radtke MA, Zschocke I, et al. The patient benefit index: a novel approach in patient- defined outcomes measurement for skin diseases. Arch Dermatol Res. 2009;301(8):561-71.

23. Feuerhahn J, Blome C, Radtke M, Augustin M. Validation of the patient benefit index for the assessment of patient-relevant benefit in the treatment of psoriasis. Arch Dermatol Res. 2012;304(6): 433-41.

24. Bharmal M, Payne K, Atkinson MJ, Desrosiers MP, Morisky DE, Gemmen E. Validation of an abbreviated Treatment Satisfaction Questionnaire for Medication (TSQM-9) among patients on antihypertensive medications. Health Qual Life Outcomes. 2009;7:36.

25. Roblin D, Wickramasinghe R, Yosipovitch G. Pruritus severity in patients with psoriasis is not correlated with psoriasis disease severity. J Am Acad Dermatol. 2014;70(2):390-1.

26. Heydendael VM, de Borgie CA, Spuls PI, Bossuyt PM, Bos JD, de Rie MA. The burden of psoriasis is not determined by disease severity only. J Investig Dermatol Symp Proc. 2004;9(2):131-5.

27. Blome C, Gosau R, Radtke MA, et al. Patient-relevant treatment goals in psoriasis. Arch Dermatol Res. 2016;308(2):69-78.

28. Maul JT, Navarini AA, Sommer R, et al. Gender and age significantly determine patient needs and treatment goals in psoriasis-a lesson for practice. J Eur Acad Dermatol Venereol. 2019;33(4):700-8. 